\title{
The network effects of railway investments
}

\author{
S. Hansen ${ }^{1}$, A. Landex ${ }^{2}$ \& A. H. Kaas ${ }^{1}$ \\ ${ }^{1}$ Atkins Danmark A/S, Denmark \\ ${ }^{2}$ Centre for Traffic and Transport, Technical University of Denmark, \\ Denmark
}

\begin{abstract}
Network effects are when a change at one place in the railway network results in changes elsewhere in the network - maybe even far away from the original change. Railway investments have network effects, and therefore, this paper describes the network effects and how these network effects can be examined by queuing time. This paper gives examples of network effects and describes the importance of the size of the analysis area and the connections between trains in the railway network.
\end{abstract}

Keywords: network effects, queuing time, timetabling.

\section{Introduction}

The aim of many railway investment projects in Europe is to remove bottlenecks in the infrastructure, thereby making it possible to reduce travel times and increase the number of trains. The size of the benefits depends of the actual project involved. In any case, it is an important planning task to determine the future timetable. This makes is possible to determine the benefits for travellers in a standard cost-benefit analysis (CBA). The scope of this paper is to discuss the influence of the size of the analysis area on the calculated travel times.

Now, once a project is completed it is fairly straightforward to recognize that the project will also influence the future timetable outside the project area, thereby creating benefits outside the local area of the project. This is denoted "Network effects" in this paper. It is not possible to claim this as a general effect.

On a rural branch-line, where local trains connect to the national InterCity system, it is unlikely that any project will influence the national timetable. The benefits on the rural branch-line will be purely local, while removing a major bottleneck on the main-line network is likely to create changes on many 
connecting lines, and maybe improve the timetable across the national network. In this case, a local analysis is not sufficient to capture all benefits.

\subsection{Network effects in the Danish context}

An example to illustrate the network effects is the Danish railway line between Aalborg and Frederikshavn, cf. figure 1. It is a single track line with a one-hour service. The travel time in one direction is 63 minutes and 66 minutes in the other direction. The speed on the line is increased from $120 \mathrm{~km} / \mathrm{h}$ to $180 \mathrm{~km} / \mathrm{h}$.

This project can be evaluated locally. However, the traffic in the northern part of Denmark is not timetabled independently of the remaining network. The trains are part of the nationwide IC system and are therefore adapted to the arrival and departure times of the IC-trains at Aalborg (as well as the crossing possibilities in the northern part of Denmark).

If the crossing in the candidate timetable for the upgrading project is moved to obtain benefits locally, e.g. 10 minutes for one of the directions, it would result in nationwide changes. It is due to the fact that most regional trains have connection to and from IC-trains. A change in the northern part of Denmark will therefore influence the regional trains Copenhagen-Nykøbing F (in the southern part of Denmark) because of the connection at Ringsted cf. figure 1. This change may very well result in time benefits (or losses) at other lines of the network.

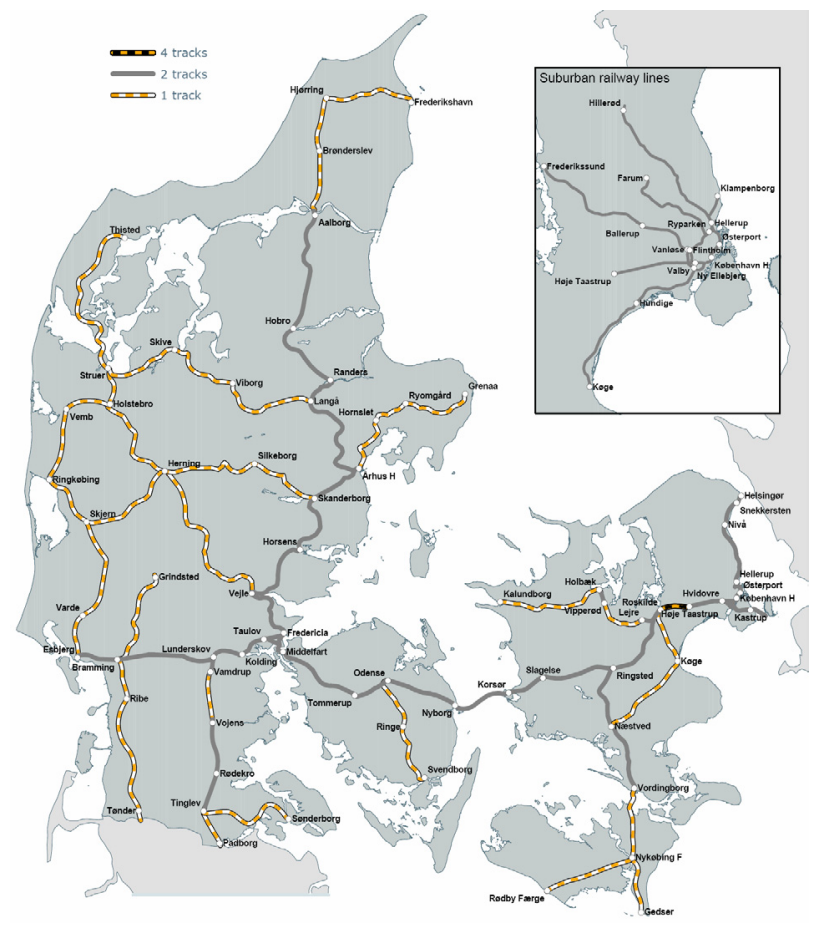

Figure 1: The Danish railway infrastructure. 
Since network effects can influence the entire railway network, the extent of which are impossible to examine, it is necessary to delimit an area of analysis. Therefore, it is interesting to examine the importance of these network effects.

\section{Methodical overview}

To identify the network effects of the above mentioned increased speed project, a nationwide candidate timetable for one standard hour must be worked out. However, the nationwide timetable in Denmark depends on the train services to/from Germany and Sweden. To evaluate all the network effects it is therefore not enough to create a nationwide candidate timetable. It is necessary to include the trains to/from Germany and Sweden and thereby also the nationwide timetables of Germany and Sweden and so forth.

Network effects can be illustrated by queuing time. Queuing time is the difference in running time when comparing a single train on a line with a situation with many trains on the line. Queuing time on railway lines occurs when the traffic intensity is close to the capacity level due to e.g. mixed operation (slow and fast trains). When close to the capacity level, the operation speeds of fast trains must/will adapt to the slower trains cf. figure 2. This will increase the travel time for the trains that under free conditions could run at higher speeds [6].

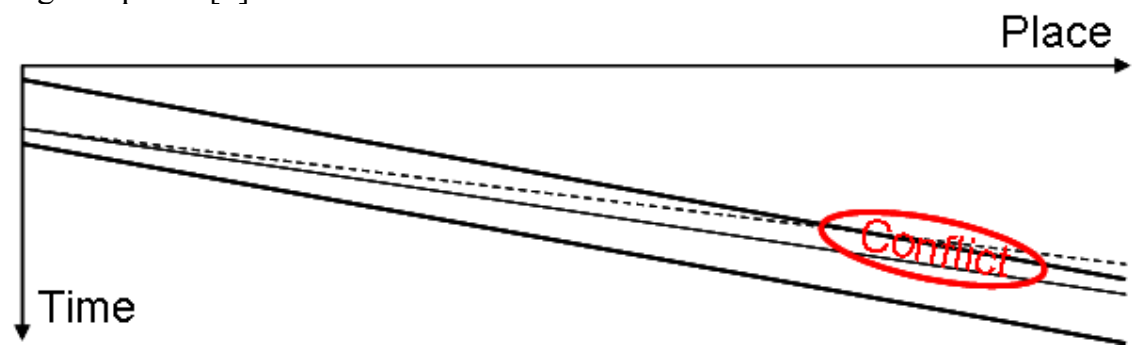

Figure 2: Extended running time (queuing time) due to other trains on the line [6].

To calculate the queuing time the Danish developed SCAN model (Strategic Capacity Analysis of Network) can be used (a similar function is found in the German tool UX-SIMU). SCAN is a computer tool for calculation of capacity in a railway network. In SCAN capacity is measured as average queuing time in a sample of candidate timetables for a given infrastructure alternative. The tool can be used in the strategic planning process where the exact infrastructure and timetable are not determined. Therefore the system is based on a structure where it is only necessary to know the plan of operation (i.e. the number of trains within each category), the infrastructure in a simple way and the main dynamics of the rolling stock [3]. 


\subsection{The general problem of network effects}

One of the problems you have to handle when calculating the consequences of a project is the size of the analysis area. When the analysis area is large, the risk of network effects is high. This is due to the fact that when a large analysis area is examined it is because of bigger changes in the infrastructure and/or timetables. Major changes in the infrastructure and/or timetables may influence many trains in the analysis area, and these trains may influence other trains outside the analysis area.

However, even smaller analysis areas may generate network effects. This is due to the way of planning the timetable in Denmark and many other countries. All train services can be placed in a hierarchy, cf. figure 3, where the train services placed in the top of the hierarchy is planned and timetabled before trains further down in the hierarchy.

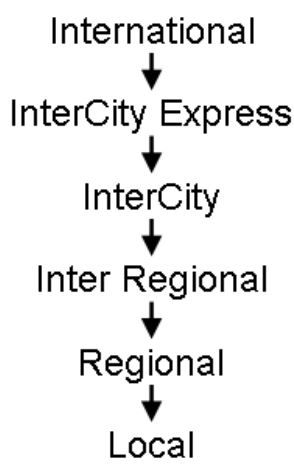

Figure 3: The hierarchy of the train service.

Even small changes in the timetable of a train in the upper level of the hierarchy may influence other trains further down in the hierarchy, because these trains are planned according to the train high up in the hierarchy. Since trains high up in the hierarchy often travel long distances, the changes for other train services can occur far away from the analysis area.

These changes in the railway network can occur far away from the analysis area is due to the network effects. Therefore, it is important to examine whether there are network effects. Nonetheless, the effects are normally only studied locally. It can be due to lack of resources, or because the network effects are uncertain (or insignificant), or because you only wish to evaluate the project locally, isolated from the remaining network.

\subsection{Network effects on the railway line between Copenhagen and Ringsted}

An example of a local study is found in the Copenhagen-Ringsted study where it was examined whether to extend the existing railway line or build an extra railway line in another layout. The capacity analysis for each of the alternatives only covers the actual line Copenhagen-Ringsted. For instance, the capacity 
conditions at Copenhagen Central Station have not been included. The analysis itself consists of calculated queuing time for basis and 3 main alternatives as well as a variant of the new construction solution see figure 4 . The analysis can be used to rank the alternatives examined, but it does not provide information about the conditions of the project alternative, when the capacity conditions on the adjacent lines are included.

\section{Queuing times found by simulation}

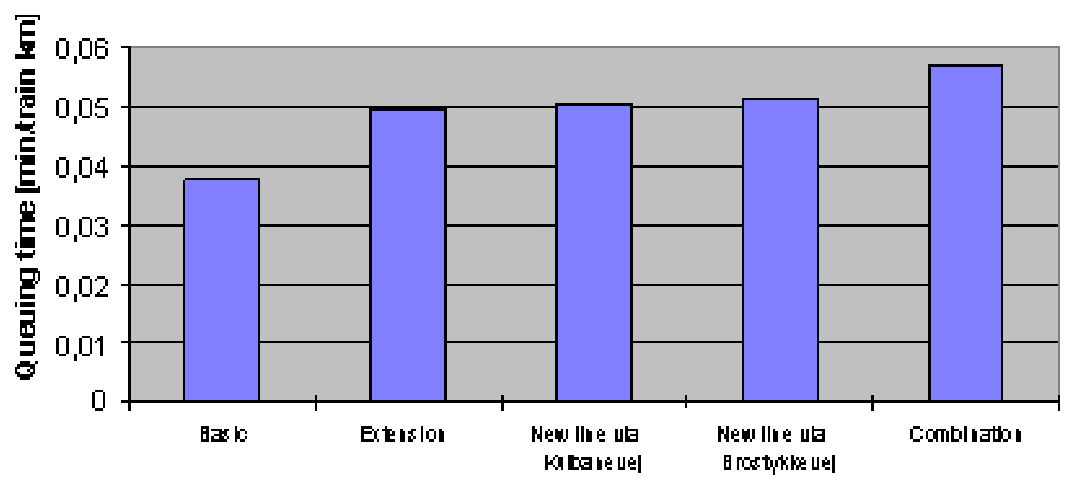

Figure 4: Queuing time analysis of the Copenhagen-Ringsted alternatives

However, when an infrastructure project is carried out, the traffic on the line or station in question is of course not planned independently of the remaining network. As the yearly timetable is planned at the network level, the timetable for the project area will also be influenced by this. The extent of this influence depends entirely on local conditions. In case of major improvements of the infrastructure on the main lines, it influences the timetable for the entire country (due to connections between long distance trains and regional trains).

\subsection{Methodical results}

The influence is bilateral, partly because a candidate timetable covering the project area may have derived effects at the network level, but the opposite can also be the case. If a candidate timetable is only worked out locally, it is not examined whether this timetable has conflicts outside the area. At worst, the candidate timetable in question gives rise to conflicts on the adjacent lines in the immediate area. Therefore, it can not be implemented in real life because of capacity conditions on the remaining network and it thus overestimates the benefits of the project.

The problem will be most significant, if the project is adjacent to lines with dense traffic/high capacity utilization. When preparing the timetables you normally start with the lines forming bottlenecks within the network, and if the 
project is adjacent to such a bottleneck, the timetable for this line will determine the timetable for the project area.

A way to recognize the network effects is to work out a candidate timetable for a bigger part of the network than the project area. The size of the network to be included will depend on the project (and the analysis level). This method is used in the Netherlands where the network is more complex than in Denmark. Here a candidate timetable for one standard hour is worked out for the entire network. This is done by the Dutch tool DONS [5]. The timetable is then used to evaluate a project, so that it reflects both local effects and network effects.

It is a methodical problem that network effects cannot be generalized. Analyses of queuing time can be used for an initial evaluation of the network effects. By first carrying out an analysis of the queuing time locally and then an analysis including the adjacent lines the difference can indicate the problem. If the level of queuing time increases (queuing time per train-km) it is necessary to look at the network effects, but if the level decreases they can (probably) be ignored. This method is tested in the following section.

\section{Network effects illustrated by queuing time}

In this section the size of the network effects is examined for the CopenhagenRingsted example by means of the queuing time method. After the public hearing in 1998, a new examination of the project was initiated in 1999, including the development of a new traffic model. For the alternatives to be examined, a set of candidate timetables were prepared.

Three of these alternatives have been selected and used in this section. With regard to the infrastructure, the alternatives are as follows:

Table 1: $\quad$ Scenarios examined.

\begin{tabular}{|l|l|}
\hline Scenario & Description of scenario \\
\hline Basis & Infrastructure in Funen and Zealand as in 1999 \\
\hline Extending line & $\begin{array}{l}\text { Two extra tracks between Copenhagen-Høje Taastrup and } \\
\text { Roskilde-Ringsted and higher speed on the line }\end{array}$ \\
\hline New Line & $\begin{array}{l}\text { Two extra tracks between Copenhagen and Ringsted and } \\
\text { higher speed on the line }\end{array}$ \\
\hline
\end{tabular}

The candidate timetables comprise the railway network in Zealand (except from the railway line between Elsinore and Copenhagen Airport) and Funen and include the line Copenhagen Central Station-Fredericia (just west of Funen), cf. figure 1 and figure 5 .

With respect to this paper these timetables have been reduced to plans of operations by eliminating all arrival and departure times, and the queuing time is then simulated in the tool UX-SIMU. In this model, queuing time is calculated as the average of all "timetables". The result is minutes of queuing time per train-km. 


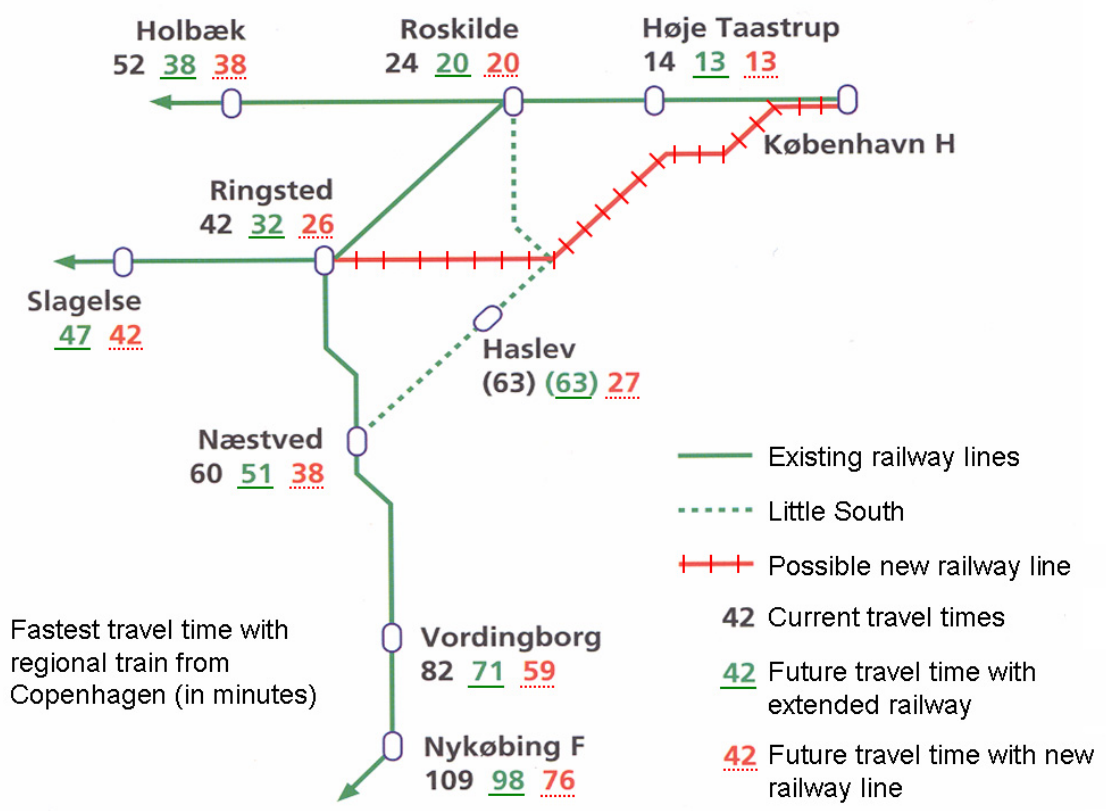

Figure 5: Fastest travel time with regional train from Copenhagen [4].

Two studies of network effects are carried out:

1. The importance of the size of the analysis area

2. The importance of including connections

In the first calculation the analysis area is reduced to include only the project area locally. The queuing time is calculated for the same trains, but only on this line, so that it is only the capacity conditions on the approximately $60 \mathrm{~km}$ long line that influence the queuing time.

In the other study the original analysis area is maintained, but now 2 connections are included in the calculation of the queuing time. A demand for connection will reduce the number of possible candidate timetables and give a more realistic calculation. Traditional analyses of queuing time do not cover this, and it is an example of an obvious calculation simplification. It is not necessarily clear which transfers there will be between the trains in a future scenario, it is however taken for granted that there will be connections somewhere in the network. The need for resources is of course smaller if the connections are ignored, and for capacity analyses, where several projects have to be ranked, it is probably acceptable.

\section{The importance of the size of the analysis area}

The importance of the analysis area has been illustrated by calculating the queuing time for the whole of East Denmark as opposed to only calculating the 
queuing time for the line that is extended, i.e. the Copenhagen-Ringsted line. In East Denmark the capacity conditions Copenhagen-Fredericia (approx. $200 \mathrm{~km}$ ) are included as well as the adjacent lines in Zealand where also single track lines are included.

The result is made up as average queuing time per train-km, as the 3 main alternatives do not include the same number of train departures. The result appears from table 2 . The average queuing time for a train that covers a distance of $100 \mathrm{~km}$ is for instance 4.2 minutes in the basis scenario.

Table 2: Queuing time in the Copenhagen-Ringsted project.

\begin{tabular}{|l|c|c|c|}
\hline \multirow{2}{*}{ Area } & Basis & Extending line & New line \\
\cline { 2 - 4 } & \multicolumn{3}{|c|}{ (Queuing time in minutes per train-km) } \\
\hline East Denmark & 0.042 & 0.048 & 0.057 \\
\hline Copenhagen-Ringsted & 0.033 & 0.046 & 0.033 \\
\hline Ratio of analysis area & $79 \%$ & $96 \%$ & $58 \%$ \\
\hline
\end{tabular}

In both Basis and Full extension (New line) scenarios it is seen that the queuing time drops considerably, if it is only calculated locally as opposed to a bigger part of the network. An isolated local examination will therefore underestimate the queuing time when the project is seen in connection with the remaining net. In New line, with 4 tracks to Ringsted, it reflects that the bottlenecks are now found on lines further away than Copenhagen-Ringsted. In the extending line scenario the amount of queuing time is more or less the same, so in this case there is a balance between traffic and infrastructure.

The figures of queuing time indicate that the capacity conditions are underestimated when you only look at the effects locally. It can also be interpreted in connection with the timetables and the travel times indicated in these. If the candidate timetable only comprises the line locally, then the possible travel times are overestimated. When the remaining part of the network is included, it results in an increase of the queuing time. In other words, travel times, as a consequence of the project, will be longer than shown by a local calculation of the travel times Copenhagen-Ringsted. Once completed, locally the timetable will depend on the remaining network.

Furthermore, when comparing the queuing time for the entire network in the three alternatives it is seen that both extension alternatives have relatively higher traffic intensity than basis. It can be interpreted in such a way that the extensions are trafficked too heavily as compared to basis; i.e. the regularity will be relatively worse than at basis (i.e. the socio-economic benefits of the projects are overestimated).

\section{The importance of connections}

In many (Danish) cases analyses of queuing time do not take transfers into consideration. To examine the importance of this simplification, it has been 
examined how much the above calculated queuing time for the whole of East Denmark is influenced, if a number of connections are taken into consideration.

The calculation has been made with 2 commonly occurring connections: At Ringsted between an IC-train and a regional train Copenhagen-Nykøbing F and at Roskilde between an IC-train and a regional train Copenhagen-Holbæk (cf. figure 1). The calculation has only been made for the extension solution, the new line scenario.

Table 3: Queuing time Copenhagen-Ringsted with connections.

\begin{tabular}{|l|c|c|c|}
\hline \multirow{2}{*}{ Area } & Basis & Extending line & New line \\
\cline { 2 - 4 } & \multicolumn{2}{|c|}{ (Queuing time in minutes per train-km) } \\
\hline East Denmark & 0.042 & 0.048 & 0.057 \\
\hline $\begin{array}{l}\text { Including connections at } \\
\text { Roskilde and Ringsted }\end{array}$ & - & - & 0.061 \\
\hline
\end{tabular}

It appears from table 3 that the amount of queuing time increases a little in the New line scenario. However, as only very few of the trains examined are subject to connection demands, it requires further studies to make a general statement.

On the other hand it can be concluded that network effects can have a big influence on the locally obtained travel times, and thereby on the calculated socio-economic benefit. As a principal rule, candidate timetables are worked out for an area that is larger than the project itself to include the effect of the capacity conditions on the adjacent lines. As a supplement to this a sensitivity analysis can be carried out by means of a local timetable. In a number of cases the local timetable will give shorter travel times and a better socio-economic profit and illustrate the effect of bottlenecks in the adjacent network.

\section{Conclusions}

This paper has presented network effects and a method to evaluate the network effects by examining the queuing time in the timetable. It has furthermore been shown that network effects are likely to occur in a railway system.

Changes in the timetable at one place of the railway network can lead to changes in the timetable for train services and/or railway lines far away. These changes on other train services and/or railway lines far away are due to network effects. Network effects are most likely to occur if changes in e.g. the infrastructure in a large analysis area are examined or if a train high up in the hierarchy of train services is influenced by the analysis.

\section{References}

[1] Hansen, S., Large Transport Infrastructure Investments and their Strategic Impacts with a Special Focus on Enterprises, PhD. thesis at Centre for Traffic and Transport, Technical University of Denmark, 2004 (in Danish). 
[2] Kaas, A. H., Methods to calculate capacity of railways, PhD. thesis at Department of Planning, Technical University of Denmark, 1998 (in Danish).

[3] Kaas, A. H., Developing and practical use of a capacity model for railway networks, Proc. of the $2^{\text {nd }}$ International Conference on Computer Applications in Railway Systems, 1998

[4] The Danish Ministry of Transport, Upgrading the main railway lines Baneplansudvalget, April 1997 (in Danish).

[5] Odijk, M. A., Railway Timetable Generation, PhD. thesis at Deft University of Technology, 1998

[6] Salling, K. B. \& Landex, A., Computer based ex-ante evaluation of the planned railway line between Copenhagen and Ringsted by use of a new Decision Support System named COSIMA-DSS, Proc. of the $10^{\text {th }}$ International conference on Computers in Railways, eds. J. Allan, C.A. Brebbia, A.F. Rumsey, G. Sciutto, S. Sone \& C.J. Goodman, 2006 